Pacific

Journal of

Mathematics

FINITE QUOTIENTS OF THE ALGEBRAIC FUNDAMENTAL GROUP OF PROJECTIVE CURVES IN POSITIVE CHARACTERISTIC

Amílcar Pacheco and Katherine F. Stevenson

Volume 192 No. 1

January 2000 


\title{
FINITE QUOTIENTS OF THE ALGEBRAIC FUNDAMENTAL GROUP OF PROJECTIVE CURVES IN POSITIVE CHARACTERISTIC
}

\author{
Amílcar Pacheco and Katherine F. Stevenson
}

Let $X$ be a smooth connected projective curve defined over an algebraically closed field $k$ of characteristic $p>0$. Let $G$ be a finite group whose order is divisible by $p$. Suppose that $G$ has a normal $p$-Sylow subgroup. We give a necessary and sufficient condition for $G$ to be a quotient of the algebraic fundamental group $\pi_{1}(X)$ of $X$.

\section{Introduction.}

Let $X$ be a smooth projective connected algebraic curve of genus $g$ defined over an algebraically closed field $k$ of characteristic $p>0$. In this paper we study necessary and sufficient conditions for a finite group $G$ to be a quotient of the algebraic fundamental group $\pi_{1}(X)$ of $X$. We denote by $\pi_{A}(X)$ the set of isomorphism classes of finite groups which are quotients of $\pi_{1}(X)$. Recall that a group $G \in \pi_{A}(X)$ will occur as a Galois group of an étale Galois cover $Z \rightarrow X$. In this paper we will call $Z \rightarrow X$ a Galois $G$-cover.

Let $G$ be a finite group and suppose that its order is not divisible by $p$. In [Groth71, Corollary 2.12] Grothendieck showed that $G \in \pi_{A}(X)$ if and only if $G$ is a quotient of the topological fundamental group $\Gamma_{g}$ of a compact Riemann surface of genus $g$.

We consider next a finite $p$-group $G$. Denote by $\Phi(G)=[G, G] G^{p}$ its Frattini subgroup and let $\mathcal{G}=G / \Phi(G)$. This group is an elementary $p$ abelian group. The $p$-torsion subgroup $J_{X}[p]$ of the Jacobian variety $J_{X}$ of $X$

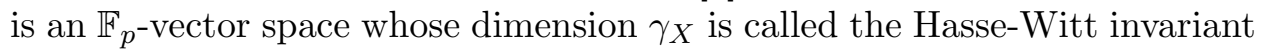
of $X$. It follows from [Ser56, $\S 11]$ that $\mathcal{G} \in \pi_{A}(X)$ if and only if $\mathcal{G}$ has $p$-rank at most $\gamma_{X}$. Suppose now that $G \in \pi_{A}(X)$, then $\mathcal{G} \in \pi_{A}(X)$, therefore the $p$-rank of $G$ (the minimal number of generators of its maximal $p$-quotient) is at most $\gamma_{X}$. Actually, this condition is also sufficient. This follows from the fact that the $p$-cohomological dimension $\operatorname{cd}_{p}\left(\pi_{1}(X)\right)$ of $\pi_{1}(X)$ is at most 1 (cf. end of proof of Theorem 1.3).

Now these two situations are understood, the next step to study is the case of a finite group $G$ whose order is divisible by $p$. Consider the case where $G$ has a normal $p$-Sylow subgroup $P$. Let $H=G / P$. The main 
theorem (Theorem 1.3) addresses the question of when a Galois $P$-cover $Z \rightarrow Y$ and a Galois $H$-cover $Y \rightarrow X$ can be composed to give a Galois $G$ cover $Z \rightarrow X$ (recall that in general the cover may not be Galois). Roughly speaking the theorem says that $G \in \pi_{A}(X)$ if and only if the action of $H$ on $P$ is compatible with the action of $H$ on $J_{Y}[p]$. The result fits nicely with the fact (implied above) that the $p$-torsion of the Jacobian variety of $Y$ regulates the Galois $P$-covers of $Y$. In order to state the main theorem precisely we need to introduce some notation.

1.1. Group theory. Let $G$ be a finite group with normal $p$-Sylow subgroup $P$ and quotient $H=G / P$. A theorem of Schur and Zassenhaus assures that $G$ is isomorphic to the semi-direct product $P \rtimes H$ taken with respect to the the action $\eta: H \rightarrow \operatorname{Aut}(P)$ defined by conjugation. Let $\Phi(P)=[P, P] P^{p}$ be the Frattini subgroup of $P$. The quotient $\mathcal{P}=P / \Phi(P)$ is the maximal elementary abelian quotient of $P$, hence it is an $\mathbb{F}_{p}$-vector space. This action induces an $\mathbb{F}_{p}$-representation $\rho: H \rightarrow \operatorname{Aut}(\mathcal{P})$.

Let $Z(H)$ be the set of irreducible characters $\chi$ of $H$ with values in the algebraically closed field $k$ of characteristic $p>0$. Let $\chi^{0}$ be the trivial character of $H$ and $\rho_{\chi}: H \rightarrow \mathrm{GL}\left(V_{\chi}\right)$ an irreducible $k$-representation of $H$ of character $\chi$ of degree $n_{\chi}$. The canonical decomposition of $\mathcal{P} \otimes_{\mathbb{F}_{p}} k$ as a $k[H]$-module is given by

$$
\mathcal{P} \otimes_{\mathbb{F}_{p}} k=\bigoplus_{\chi \in Z(H)} V_{\chi}^{m_{\chi}}
$$

1.2. Generalized Hasse-Witt invariants. Let $Y \rightarrow X$ be a Galois cover with $\operatorname{Gal}(Y / X) \cong H$ and $g_{Y}$ the genus of $Y$. Let $J_{Y}$ be the Jacobian variety of $Y$ and $J_{Y}[p]$ its $p$-torsion subgroup. Suppose that $\mathbb{F}_{q}=\mathbb{F}_{p^{m}}$ is a finite field large enough to contain the $|H|$-th roots of unity. Let $e_{\chi}=$ $\frac{\chi(1)}{|H|} \sum_{h \in H} \chi\left(h^{-1}\right) h \in k[H]$ be the idempotent corresponding to $\chi$. Denote by

$$
J_{Y}[p] \otimes_{\mathbb{F}_{p}} \mathbb{F}_{q}=\bigoplus_{\chi \in Z(H)} J_{Y}[p]_{\chi}
$$

the canonical decomposition of $J_{Y}[p] \otimes_{\mathbb{F}_{p}} \mathbb{F}_{q}$, where $J_{Y}[p]_{\chi}=e_{\chi} \cdot\left(J_{Y}[p] \otimes_{\mathbb{F}_{p}}\right.$ $\mathbb{F}_{q}$ ).

Definition 1.1. The generalized Hasse-Witt invariant $\gamma_{Y, \chi}$ of $Y$ with respect to $\chi$ is defined as the dimension of $J_{Y}[p]_{\chi}$ as an $\mathbb{F}_{q}$-vector space (cf. [Ruc86, §2]). A surjection $\phi: \pi_{1}(X) \rightarrow H$ corresponds to a Galois $H$-cover $Y \rightarrow X$, and in the case that the cover $Y \rightarrow X$ is not named, we will use $\gamma_{\phi, \chi}$ to denote the generalized Hasse-Witt invariant $\gamma_{Y, \chi}$ of $Y$ with respect to $\chi$.

The notation $\gamma_{\phi, \chi}$ has the advantage that it emphasizes that the generalized Hasse-Witt invariants are invariants of the cover $Y \rightarrow X$ (corresponding 
to the surjection $\left.\phi: \pi_{1}(X) \rightarrow H\right)$ rather than of the curve $Y$ alone. Also, the main result of this paper is phrased in terms of embedding problems involving $\phi$. Thus, the notation $\gamma_{\phi, \chi}$ eases the exposition in that case. However, in the literature the notation $\gamma_{Y, \chi}$ is standard. Moreover, in this paper when we are dealing directly with the cover $Y \rightarrow X$, as opposed to the surjection $\phi$, we use the notation $\gamma_{Y, \chi}$.

A consequence of (1.2) is

$$
\gamma_{Y}=\sum_{\chi \in Z(H)} \gamma_{Y, \chi}
$$

\subsection{Embedding problems.}

Definition 1.2 ([Har95, p. 366]). An embedding problem for a profinite group $\Lambda$ is a pair of surjective profinite group homomorphisms $\left(\alpha: \Lambda \rightarrow \mathcal{K}_{2}\right.$, $\left.\delta: E \rightarrow \mathcal{K}_{2}\right)$. The embedding problem is finite, if $E$ is a finite group, and trivial, if $\delta$ is an isomorphism. A weak, respectively proper, solution to the embedding problem is a homomorphism, respectively a surjective homomorphism, $\beta: \Lambda \rightarrow E$ such that $\alpha=\delta \circ \beta$.

1.4. Main theorem. Here we give the statement of the main result. Since a surjection $\phi: \pi_{1}(X) \rightarrow H$ corresponds to a unique Galois $H$-cover $Y \rightarrow X$, embedding problems $\left(\phi: \pi_{1}(X) \rightarrow H, G \rightarrow H\right)$ relate to Galois theory. Specifically, a proper solution to such an embedding problem corresponds to the existence of a Galois $G$-cover $Z \rightarrow X$ dominating the Galois $H$-cover $Y \rightarrow X$. Thus we use the language of embedding problems to state the main theorem.

Again we assume throughout this paper that all curves are smooth connected projective $k$-curves. For such a curve $X$ we make the following notation.

Notation. Given a group $G$ with normal $p$-Sylow subgroup $P$ and quotient $H=G / P$, and given $\phi: \pi_{1}(X) \rightarrow H$, let $m_{\chi}, n_{\chi}$, and $\gamma_{\phi, \chi}$ be as in Sections 1.1 and 1.2. By Condition $A$ for the curve $X$ we will mean that for every $\chi \in Z(H)$ the following inequality holds: $m_{\chi} n_{\chi} \leq \gamma_{\phi, \chi}$.

Theorem 1.3. Let $G$ be a finite group having a normal $p$-Sylow subgroup $P$. Let $H=G / P$. An embedding problem $\left(\phi: \pi_{1}(X) \rightarrow H, G \rightarrow H\right)$ has a proper solution if and only if Condition $A$ holds for the curve $X$.

The necessity of Condition A for the curve $X$ in Theorem 1.3 was previously obtained in [Ste96a, Proposition 3.4]. In this paper we show that it is also sufficient. Rephrasing this in terms of covers we obtain the following immediate corollary.

Corollary 1.4. Let $G$ be a finite group having a normal $p$-Sylow subgroup $P$. Let $H=G / P$. Then, $G \in \pi_{A}(X)$ if and only if there exists a Galois $H$-cover $Y \rightarrow X$ such that $m_{\chi} n_{\chi} \leq \gamma_{Y, \chi}$, for every $\chi \in Z(H)$. 
Remark 1.5. In the case that $\phi$ corresponds to a Galois $H$-cover $Y \rightarrow X$ where $Y$ is an ordinary curve (namely, that the genus of $Y$ is equal to $\gamma_{Y}$ ) we show (Theorem 7.1) that an embedding problem $\left(\phi: \pi_{1}(X) \rightarrow H, G \rightarrow H\right)$ has a proper solution if and only if $m_{\chi} \leq g$, when $\chi$ is the trivial character of $H$, and $m_{\chi} \leq(g-1) n_{\chi}$, otherwise. The advantage here is that we eliminate the generalized Hasse-Witt invariant notation from the condition. This result appears in Section 7 where we discuss it and other consequences of Theorem 1.3. The existence or non-existence of 'ordinary Galois $H$-covers' is a difficult and open problem. However, in the case that $H$ is abelian and $X$ is 'generic' (cf. Section 7) a great deal of progress has been made by Nakajima [Nak83] and Zhang [Zha92]. We use their theorems in Section 7 to obtain some interesting results and examples (cf. Theorem 7.4 and Example 7.11).

We start with some Preliminaries which allow us to compute the generalized Hasse-Witt invariants in terms of differentials and to estimate how big they are. Next, in Section 3, we determine when $\mathcal{P} \rtimes H \in \pi_{A}(X)$ and develop some elementary representation theory tools which will be used in Section 6 to prove Theorem 1.3. In Section 4, some useful results regarding solutions of embedding problems are given. In Section 5 , we prove that the $p$-cohomological dimension of $\pi_{1}(X)$ is at most 1 . The main result is proved in Section 6, and in Section 7 we discuss some consequences of the main theorem and make some comparisons to previous work of Nakajima [Nak87] and Stevenson [Ste96a].

\section{Preliminaries.}

Let $Y$ be a smooth projective connected algebraic curve of genus $g_{Y}$ defined over an algebraically closed field $k$ of characteristic $p>0$.

Definition 2.1. Let $\Omega_{Y}^{1}$ be the space of differentials of $Y$ and $\Omega_{Y}^{1}(0) \subset \Omega_{Y}^{1}$ the subspace of regular differentials. Let $L$ be the function field of $Y$ and $t$ a separating variable of $L$. Given $\omega=f d t \in \Omega_{Y}^{1}$, the Cartier operator is defined by $\mathcal{C}(\omega)=\left(-d^{p-1} f / d t^{p-1}\right)^{1 / p} d t$. This is a $1 / p$-linear operator, i.e., $\mathcal{C}\left(a^{p} \omega\right)=a \mathcal{C}(\omega)$, for any $a \in K$. Moreover, $\mathcal{C}$ acts on $\Omega_{Y}^{1}(0)$ (cf. [Ser56, $\S 10$, p. 39]).

It also follows from $\left[\right.$ Ser56, $\S 10$, p. 39] that there exists an $\mathbb{F}_{p}$-isomorphism between $J_{Y}[p]$ and $\operatorname{Ker}\left(1-\mathcal{C} \mid \Omega_{Y}^{1}(0)\right)$ given by $\operatorname{class}(D) \mapsto d f / f$, where $p \cdot \operatorname{class}(D)=\operatorname{div}(f)$. In particular, $\gamma_{Y} \leq g_{Y}$.

Definition 2.2. The curve $Y$ is called ordinary if $\gamma_{Y}=g_{Y}$.

In order to understand how big the generalized Hasse-Witt invariants are we recall that a theorem of Nakajima ([Nak84] Corollary, one can also follow the proof in characteristic 0 of Chevalley and Weil [CheWei34]) 
which says that if $Y \rightarrow X$ is étale and $\operatorname{Gal}(Y / X) \cong H$, then we have an isomorphism of $k[H]$-modules

$$
\Omega_{Y}^{1}(0) \cong k \oplus k[H]^{g-1} .
$$

Given $\chi \in Z(H)$, let $\Omega_{Y}^{1}(0)_{\chi}=e_{\chi} \cdot \Omega_{Y}^{1}(0)$ and $g_{\chi}=\operatorname{dim}_{k} \Omega_{Y}^{1}(0)_{\chi}$. Note that (2.1) implies that $g_{\chi^{0}}=g$ and $g_{\chi}=(g-1) n_{\chi}^{2}$ for every $\chi \in Z(H)$, $\chi \neq \chi^{0}$. It is a result due to Rück [Ruc86, Proposition 2.3] that the $\mathbb{F}_{q}[H]$ modules $J_{Y}[p]_{\chi}$ and $\operatorname{Ker}\left(1-\mathcal{C}^{m} \mid \Omega_{Y}^{1}(0)_{\chi}\right)$ are isomorphic (this generalizes the above result of Serre). Hence, for each $\chi \in Z(H)$ we have

$$
\gamma_{Y, \chi} \leq g_{\chi}
$$

Remark 2.3. In particular, by (1.3), we conclude that $Y$ is ordinary if and only if for each $\chi \in Z(H)$ we have

$$
\gamma_{Y, \chi}= \begin{cases}g, & \text { if } \chi=\chi^{0} \text { and } \\ (g-1) n_{\chi}^{2}, & \text { if } \chi \neq \chi^{0}\end{cases}
$$

\section{Unramified covers and Galois modules.}

Let $Y \rightarrow X$ be a Galois cover with $\operatorname{Gal}(Y / X) \cong H$ and $Z \rightarrow Y$ an étale Galois cover with $\operatorname{Gal}(Z / Y) \cong(\mathbb{Z} / p \mathbb{Z})^{r}$, for some $1 \leq r \leq \gamma_{Y}$. In [Pac95, Propositions 2.4 and 2.5] the first author determined a necessary and sufficient condition for $Z \rightarrow X$ to be also Galois. We review these results and as a consequence we obtain a necessary and sufficient condition for $\mathcal{P} \rtimes H \in \pi_{A}(X)$.

Denote by $\mathcal{S}_{1}$ the set of all étale Galois covers $Z \rightarrow Y$ with $\operatorname{Gal}(Z / Y) \cong$ $(\mathbb{Z} / p \mathbb{Z})^{r}$ for some $1 \leq r \leq \gamma_{Y}$. This set corresponds bijectively to the set $\mathcal{S}_{2}$ of $\mathbb{F}_{p}$-vector subspaces of $\operatorname{Hom}\left(\pi_{1}(Y), \mathbb{Z} / p \mathbb{Z}\right)$ by $(Z \rightarrow Y) \mapsto \operatorname{Hom}(\operatorname{Gal}(Z / Y)$, $\mathbb{Z} / p \mathbb{Z})$, where we identify $\operatorname{Hom}(\operatorname{Gal}(Z / Y), \mathbb{Z} / p \mathbb{Z})$ with the $\mathbb{F}_{p \text {-vector space }}$ of $\psi \in \operatorname{Hom}\left(\pi_{1}(Y), \mathbb{Z} / p \mathbb{Z}\right)$ such that $\pi_{1}(Z) \subset \operatorname{Ker}(\psi)$. Its inverse is equal to $V \mapsto(Z \rightarrow Y)$, where $\bigcap_{\psi \in V}\left(L^{\text {un }}\right)^{\operatorname{Ker}(\psi)}$ is the function field of $Z, L$ is the function field of $Y$ and $L^{\text {un }}$ is the maximal unramified Galois extension of $L$. An element $(Z \rightarrow Y)$ of $\mathcal{S}_{1}$ is explicitly described as follows.

For each $Q \in Y$, let $L_{Q}$ be the completion of $L$ at $Q, U_{L}=$ $\bigcap_{Q \in Y}\left(\wp\left(L_{Q}\right) \cap L\right)$, where $\wp$ denotes the operator $\wp(x)=x^{p}-x$. Let $W_{L}=U_{L} / \wp(L)$ and for each $a \in U_{L}-\wp(L)$, let $\langle a+\wp(L)\rangle$ be the cyclic subgroup of order $p$ of $W_{L}$ generated by $a+\wp(L)$. Denote by $\wp^{-1}(a)$ a solution of $\wp(T)=a$ in the algebraic closure of $L$.

Lemma 3.1 ([Pac95, Proposition 2.4]). Let $(Z \rightarrow Y) \in \mathcal{S}_{1}$ with $\operatorname{Gal}(Z / Y)$ $\cong(\mathbb{Z} / p \mathbb{Z})^{r}$ for some $1 \leq r \leq \gamma_{Y}$. There exist $\mathbb{F}_{p}$-linearly independent $a_{1}+\wp(L), \ldots, a_{r}+\wp(L) \in W_{L}$ such that $k(Z)=k\left(\wp^{-1}\left(a_{1}\right), \cdots, \wp^{-1}\left(a_{r}\right)\right)$. Moreover, the cover $(Z \rightarrow Y)$ is uniquely determined by the $\mathbb{F}_{p}$-vector subspace $A_{Z / Y}=\bigoplus_{j=1}^{r}\left\langle a_{j}+\wp(L)\right\rangle$ of $W_{L}$ and $\operatorname{Gal}(Z / Y)=\operatorname{Hom}\left(A_{Z / Y}, \mathbb{Z} / p \mathbb{Z}\right)$. 
Lemma 3.2 ([Pac95, Proposition 2.5]). With hypothesis and notation as in Lemma 3.1, $Z \rightarrow X$ is Galois if and only if $A_{Z / Y}$ is an $\mathbb{F}_{p}[H]$-module. In this case, $\operatorname{Gal}(Z / X) \cong \operatorname{Gal}(Z / Y) \rtimes H$ and the action of $H$ on $A_{Z / Y}$ is contragradient to the natural action of $H$ on $\operatorname{Gal}(Z / Y)$.

Our goal now is to describe the $\mathbb{F}_{p}[H]$-module structure of $\mathcal{P}$ and compare it with the $\mathbb{F}_{p}[H]$-module structure of $\operatorname{Ker}\left(1-\mathcal{C} \mid \Omega_{Y}^{1}(0)\right)$. In order to do this we introduce some basic facts on representation theory.

Definition 3.3. Let $\chi \in Z(H)$ and denote by $\rho_{\chi}: H \rightarrow \mathrm{GL}\left(V_{\chi}\right)$ an irreducible representation with character $\chi$. Given $h \in H$, let $\left(a_{i j}(h)\right)$ be the matrix of $\rho_{\chi}(h)$ with respect to some fixed basis of $V_{\chi}$. For each $m \geq 0$, let $\rho_{\chi^{p^{m}}}: H \rightarrow \operatorname{GL}\left(V_{\chi}\right)$ be the map defined by $\rho_{\chi^{p^{m}}}(h)=\rho_{\chi}(h)^{p^{m}}$.

Lemma 3.4 ([Isa76, p. 151]). The map $\rho_{\chi^{p^{m}}}$ is an irreducible k-representation of $H$ with character $\chi^{p^{m}}$ defined by $\chi^{p^{m}}(h)=\chi(h)^{p^{m}}$.

Definition 3.5 ([Isa76, p. 152]). Denote by $\mathbb{F}_{p^{l} \chi}$ the field $\mathbb{F}_{p}(\chi)$ generated by $\mathbb{F}_{p}$ and the character values $\{\chi(h) ; h \in H\}$. Given $\chi, \psi \in Z(H)$, define $\chi \sim \psi$ if and only if there exists $0 \leq m<l_{\chi}$ such that $\psi=\chi^{p^{m}}$. Let $[\chi]$ be the class of $\chi$ in $\mathcal{Z}(H)=Z(H) / \sim$. Let $\mathcal{F}$ be the set of $\mathbb{F}_{p^{-}}$irreducible representations $\rho: H \rightarrow \mathrm{GL}(U)$ of $H$.

Lemma 3.6 ([Isa76, Theorem 9.21]). There is a bijection between the sets $\mathcal{F}$ and $\mathcal{Z}(H)$ given by $\rho \longmapsto[\chi]$, where $\rho \otimes_{\mathbb{F}_{p}} k: H \rightarrow \operatorname{GL}\left(U \otimes_{\mathbb{F}_{p}} k\right)$ is isomorphic to $\rho_{[\chi]}=\bigoplus_{j=0}^{l_{\chi}-1} \rho_{\chi^{p^{j}}}$.

The action $\eta: H \rightarrow \operatorname{Aut}(P)$ given by conjugation induces an $\mathbb{F}_{p}$-representation $\rho: H \rightarrow \operatorname{Aut}(\mathcal{P})$. By Lemma 3.6, $\rho \otimes_{\mathbb{F}_{p}} k$ is a sum of the representations $\rho_{[\chi]}$ with multiplicities $m_{\chi}$ (note that since $\rho$ is defined over $\mathbb{F}_{p}, m_{\psi}=m_{\chi}$, for $\psi \sim \chi)$. Denote $V_{[\chi]}=\bigoplus_{j=0}^{l_{\chi}-1} V_{\chi^{p^{j}}}$. Hence,

$$
\mathcal{P} \otimes_{\mathbb{F}_{p}} k \cong \bigoplus_{[\chi] \in \mathcal{Z}(H)} V_{[\chi]}^{m_{\chi}} .
$$

Let $\mathcal{V}_{[\chi]}$ be the irreducible $\mathbb{F}_{p}[H]$-module such that $\mathcal{V}_{[\chi]} \otimes_{\mathbb{F}_{p}} k \cong V_{[\chi]}$. It follows from (3.1) that

$$
\mathcal{P} \cong \bigoplus_{[\chi] \in \mathcal{Z}(H)} \mathcal{V}_{[\chi]}^{m_{\chi}}
$$

Let $n_{\chi}=\operatorname{dim}_{k} V_{\chi}, g_{\chi}=\operatorname{dim}_{k} \Omega_{Y}^{1}(0)_{\chi}$ and $\Omega_{Y}^{1}(0)_{[\chi]}=\bigoplus_{i=0}^{l_{\chi}-1} \Omega_{Y}^{1}(0)_{\chi^{p^{i}}}$. The Cartier operator $\mathcal{C}$ induces a $k$-isomorphism between $\Omega_{Y}^{1}(0)_{\chi^{p}}$ and $\Omega_{Y}^{1}(0)_{\chi}$ given by $\omega \mapsto \mathcal{C}(\omega)$. In particular, $\Omega_{Y}^{1}(0)_{[\chi]} \cong V_{[\chi]}^{g_{\chi} / n_{\chi}}$. Clearly $\mathcal{C}$ acts on $\Omega_{Y}^{1}(0)_{[\chi]}$. Hence, $\operatorname{Ker}\left(1-\mathcal{C} \mid \Omega_{Y}^{1}(0)_{[\chi]}\right) \cong \mathcal{V}_{[\chi]}^{t_{\chi}}$, for some $1 \leq t_{\chi} \leq g_{\chi} / n_{\chi}$. 
The canonical decomposition of $\Omega_{Y}^{1}(0)$ into irreducible $k[H]$-modules is given by

$$
\Omega_{Y}^{1}(0)=\bigoplus_{\chi \in Z(H)} \Omega_{Y}^{1}(0)_{\chi}=\bigoplus_{[\chi] \in \mathcal{Z}(H)} \Omega_{Y}^{1}(0)_{[\chi]} .
$$

As a consequence we obtain the canonical decomposition

$$
\begin{aligned}
\operatorname{Ker}\left(1-\mathcal{C} \mid \Omega_{Y}^{1}(0)\right) & =\bigoplus_{[\chi] \in \mathcal{Z}(H)} \operatorname{Ker}\left(1-\mathcal{C} \mid \Omega_{Y}^{1}(0)_{[\chi]}\right) \\
& \cong \bigoplus_{[\chi] \in \mathcal{Z}(H)} \mathcal{V}_{[\chi]}^{t_{\chi}}
\end{aligned}
$$

of $\operatorname{Ker}\left(1-\mathcal{C} \mid \Omega_{Y}^{1}(0)\right)$ into irreducible $\mathbb{F}_{p}[H]$-modules.

\section{Cohomological dimension and embedding problems.}

In this section we describe one tool from Galois cohomology which we use to prove that if $\mathcal{P} \rtimes H \in \pi_{A}(X)$ and $\operatorname{cd}_{p}\left(\pi_{1}(X)\right) \leq 1$, then $G \in \pi_{A}(X)$. This result is expressed in terms of embedding problems (cf. Remark 4.4). This concept is also reviewed here.

Definition 4.1 ([Ser86, I-17]). A profinite group $\Lambda$ has $p$-cohomological dimension at most $d \geq 1$, if for every $\Lambda$-module $M$ and for every integer $e>d$ the $p$-primary component of $H^{e}(\Lambda, M)$ is trivial. The infimum $\operatorname{cd}_{p}(\Lambda)$ of all such $d$ is called the $p$-cohomological dimension of $\Lambda$.

Definition 4.2 ([Ser86, I-23, 3.4]). Let

$$
1 \rightarrow \mathcal{K}_{1} \rightarrow E \stackrel{\delta}{\rightarrow} \mathcal{K}_{2} \rightarrow 1
$$

be an extension of profinite groups. A profinite group $\Lambda$ has the lifting property for this extension, if for every homomorphism $\alpha: \Lambda \rightarrow \mathcal{K}_{2}$ there exists a homomorphism $\beta: \Lambda \rightarrow E$ such that $\alpha=\delta \circ \beta$.

Proposition 4.3 ([Ser86, Proposition 16, I-23]). The inequality $\operatorname{cd}_{p}(\Lambda) \leq 1$ holds if and only if the extension (4.1) has the lifting property, when $\mathcal{K}_{1}$ is a pro-p group.

Remark 4.4. In the case where $\operatorname{cd}_{p}(\Lambda) \leq 1$, it follows from Proposition 4.3 and Definition 1.2 that there exists a weak solution to the embedding problem

$$
\left(\delta: E \rightarrow \mathcal{K}_{2}, \Lambda \rightarrow \mathcal{K}_{2}\right) .
$$

Let $G$ be a finite group having a normal $p$-Sylow subgroup $P, H=G / P$ and $\mathcal{P}=P / \Phi(P)$. Recall that $G \cong P \rtimes H$. Define $\delta_{G}: G \rightarrow \mathcal{P} \rtimes H$ by $\delta_{G}((a, b))=$ $(a \bmod \Phi(P), b)$. This function is a surjective group homomorphism and $\operatorname{Ker}\left(\delta_{G}\right)=\Phi(P)$. 
In particular, if $\operatorname{cd}_{p}\left(\pi_{1}(X)\right) \leq 1$ and $\mathcal{P} \rtimes H \in \pi_{A}(X)$, then there exists a weak solution $\pi_{1}(X) \rightarrow G$ to the embedding problem

$$
\left(\delta_{G}: G \rightarrow \mathcal{P} \rtimes H, \pi_{1}(X) \rightarrow \mathcal{P} \rtimes H\right) .
$$

Furthermore, this weak solution is indeed a proper one, because $\Phi(P) \subset$ $\Phi(G)$ and the latter set is exactly the set of "non-generators" of $G$, thus $\pi_{1}(X) \rightarrow G$ must be surjective.

\section{Cohomological dimension at most one.}

In this section we prove that the $p$-cohomological dimension $\pi_{1}(X)$ is at most 1. The proof follows the argument sketched out by Serre in [Ser90, Proposition 1] where he proves a similar result for an affine curve $U$ (sf. also [Kat88]).

Definition 5.1. Let $X$ be a smooth projective connected curve defined over $k$. Denote by FEt/ $X$ the category of finite étale covers of $X$. Given a closed point $\bar{x}$ of $X$ define the functor $\mathfrak{F}:$ FEt $/ X \rightarrow$ Sets by $Y \mapsto \operatorname{Hom}_{X}(\bar{x}, Y)$.

Remark 5.2. It follows from [Mil80, Chapter I, $\S 5$, p. 39] that $\mathfrak{F}$ is strictly pro-representable, i.e., there exists a projective system $\left(X_{\nu}, \phi_{\nu \mu}\right)$ in FEt/X where the transition morphisms $\phi_{\nu \mu}: X_{\nu} \rightarrow X_{\mu}$ are epimorphisms for $\nu \geq \mu$ and the elements $f_{\nu} \in \operatorname{Hom}_{X}\left(\bar{x}, X_{\nu}\right)$ satisfy

1) $f_{\nu}=\phi_{\nu \mu} \circ f_{\mu}$; and

2) for any $Y \in \mathbf{F E t} / X$ the natural map $\underline{\lim }_{\nu} \operatorname{Hom}_{X}\left(X_{\nu}, Y\right) \rightarrow \operatorname{Hom}_{X}(\bar{x}$, $Y)$ is an isomorphism.

Notation. Given a morphism $Y \rightarrow X$ and $\mathcal{F}$ an étale sheaf on $X$ (cf. [Mil80, Chapter II]), we denote by $\mathcal{F}_{\mid Y}$ the pullback of $\mathcal{F}$ to $Y$. For any $n \geq 0$ and $\alpha \in H_{\text {ét }}^{n}(X, \mathcal{F})$ denote by $\alpha_{\mid Y} \in H_{\text {ét }}^{n}\left(Y, \mathcal{F}_{\mid Y}\right)$ the pullback of $\alpha$ to $Y$.

Definition 5.3 ([Mil80, p. 155 and 220]). An étale sheaf $\mathcal{F}$ on $X$ is called finite if for every quasi-compact $U \subset X, \mathcal{F}(U)$ is finite. $\mathcal{F}$ has finite stalks if for every geometric point $\bar{x}$ of $X, \mathcal{F}_{\bar{x}}$ is finite. $\mathcal{F}$ is called locally constant if there exists a covering $\left(U_{\xi} \rightarrow X\right)_{\xi \in \Xi}$ such that for every $\xi \in \Xi, \mathcal{F}_{\mid U_{\xi}}$ is constant. $\mathcal{F}$ is called a $p$-torsion sheaf if for every quasi-compact $U \subset X$, $\mathcal{F}(U)$ is killed by a power of $p$.

Proposition 5.4 ([Mil80, Proposition 1.1, Remark 1.2 (b)]). Each locally constant sheaf $\mathcal{F}$ on $X$ with finite stalks is finite and represented by a group scheme $\widetilde{\mathcal{F}}$ that is finite and étale over $X$. Furthermore, there exists a finite étale morphism $X^{\prime} \rightarrow X$ such that $\widetilde{\mathcal{F}} \times_{X} X^{\prime}$ is a disjoint union of copies of $X^{\prime}$ and $\mathcal{F}_{\mid X^{\prime}}$ is constant. 
Convention. From this point till the end of this section, unless otherwise stated, $\mathcal{F}$ will denote a $p$-torsion locally constant sheaf on $X$ with finite stalks.

Remark 5.5. It follows from Definition 5.3 and Proposition 5.4 that

$$
\mathcal{F}_{\mid X^{\prime}} \cong \bigoplus_{i=1}^{r}\left(\mathbb{Z} / p^{n_{i}} \mathbb{Z}\right)^{m_{i}},
$$

where the $n_{i}$ 's and $m_{i}$ 's are positive integers.

Proposition 5.6. For each $Y \in \mathbf{F E t} / X$ and $\beta \in H_{\mathrm{et}}^{1}\left(Y, \mathcal{F}_{\mid Y}\right)$ there exists $Z \in \mathbf{F E t} / X$ such that $Z$ factors through $Y$ and $\beta_{\mid Z} \in H_{\mathrm{et}}^{1}\left(Z, \mathcal{F}_{\mid Z}\right)$ is trivial.

Proof. We start with the case where $Y=X$. Given $\beta \in H_{\text {et }}^{1}(X, \mathcal{F})$, let $x^{\prime}$ be as in Prop. 5.4 and $\beta^{\prime}=\beta_{\mid X^{\prime}} \in H_{\text {et }}^{1}\left(X^{\prime}, \mathcal{F}_{\mid X^{\prime}}\right)$. By $(5.1)$

$$
H_{\mathrm{et}}^{1}\left(X^{\prime}, \mathcal{F}_{\mid X^{\prime}}\right) \cong \bigoplus_{i=1}^{r} H_{\mathrm{et}}^{1}\left(X^{\prime}, \mathbb{Z} / p^{n_{i}} \mathbb{Z}\right)^{m_{i}} .
$$

So, we denote $\beta^{\prime}=\left(\beta_{1,1}, \ldots, \beta_{1, m_{1}}, \ldots, \beta_{r, 1}, \ldots, \beta_{r, m_{r}}\right)$ with $\beta_{i, j} \in H_{\text {et }}^{1}\left(X^{\prime}\right.$, $\left.\mathbb{Z} / p^{n_{i}} \mathbb{Z}\right)$. Let $\mathcal{W}_{n}$ be the sheaf of Witt vectors of length $n$ on $X$ [Ser56, $\S 2], F_{\text {abs }}: X \rightarrow X$ the absolute Frobenius morphism and $\wp$ the operator $\wp(x)=x^{p}-x$. The exact sequence

$$
1 \rightarrow \mathbb{Z} / p^{n} \mathbb{Z} \rightarrow \mathcal{W}_{n} \stackrel{\wp}{\rightarrow} \mathcal{W}_{n} \rightarrow 1,
$$

gives an isomorphism $H_{\text {et }}^{1}\left(X, \mathbb{Z} / p^{n} \mathbb{Z}\right) \cong H^{1}\left(X, \mathcal{W}_{n}\right)^{F_{\text {abs }}}$, as in the usual Artin-Schreier theory [Mil80, p. 127-128]. Hence, by [Ser56, Proposition 13], we conclude that $\beta_{i, j}$ parametrizes a cyclic étale cover $X_{i, j} \rightarrow X^{\prime}$ of degree $p^{n_{i}}$. Given $\alpha \in H_{\text {et }}^{1}\left(X^{\prime}, \mathbb{Z} / p^{n} \mathbb{Z}\right)$ and $V \rightarrow X^{\prime}$ any finite étale cover, let $X^{\prime \prime} \rightarrow X^{\prime}$ be the cyclic étale cover of degree $p^{n}$ defined by $\alpha$ and let $\alpha^{\prime}=\alpha_{\mid V} \in H_{\mathrm{et}}^{1}\left(V, \mathbb{Z} / p^{n} \mathbb{Z}_{\mid V}\right)$. Thus $\alpha^{\prime}$ parametrizes the covering $W=V \times_{X^{\prime}} X^{\prime \prime} \rightarrow V$. In the case where $\alpha=\beta_{i, j}$, the covering $X_{i, j} \rightarrow X^{\prime}$ plays the role of both $V \rightarrow X^{\prime}$ and $X^{\prime \prime} \rightarrow X^{\prime}$. Therefore $\beta_{i, j \mid X_{i, j}}$ is trivial. Let $Z \rightarrow X^{\prime}$ be a finite étale cover such that for every $i \in\{1, \ldots, r\}$ and $j \in\left\{1, \ldots, m_{i}\right\}$. The cover $Z \rightarrow X^{\prime}$ factors through $X_{i, j} \rightarrow X^{\prime}$. Therefore $\beta_{\mid Z}=\beta_{\mid Z}^{\prime} \in H_{\text {et }}^{1}\left(Z, \mathcal{F}_{\mid Z}\right)$ is trivial.

In the case where $Y \neq X$, let $Y^{\prime}=Y \times_{X} X^{\prime}$. We have

$$
\mathcal{F}_{\mid Y^{\prime}} \cong \bigoplus_{i=1}^{r}\left(\mathbb{Z} / p^{n_{i}} \mathbb{Z}\right)^{m_{i}}
$$

It follows from the above argument that there exists a finite étale cover $Z \rightarrow Y^{\prime}$ such that $\beta_{\mid Z}=\left(\beta_{\mid Y^{\prime}}\right)_{\mid Z} \in H_{\mathrm{et}}^{1}\left(Z, \mathcal{F}_{\mid Z}\right)$ is trivial.

Proposition 5.7. For each $Y \in$ FEt/ $X$ there exists $Z \in$ FEt/ $X$ which factors through $Y$ such that $H_{\mathrm{et}}^{0}\left(Z, \mathcal{F}_{\mid Z}\right) \cong \bigoplus_{i=1}^{r}\left(\mathbb{Z} / p^{n_{i}} \mathbb{Z}\right)^{m_{i}}$. 
Proof. As in the proof of Proposition 5.6 it suffices to take $Z=Y \times_{X} X^{\prime}$.

Remark 5.8 ([Mil80, Chapter I, 5.4]). Given $Y \in$ FEt/ $X$ denote by $\operatorname{Aut}_{X}(Y)$ the set of $X$-automorphisms of $Y$. There exists $Z \in \mathbf{F E t} / X$ such that $Z \rightarrow X$ is Galois and $Z \rightarrow Y$ is an $X$-morphism. In this case $\operatorname{Hom}_{X}(\bar{x}, Z)$ is isomorphic to $\operatorname{Aut}_{X}(Z)$. In particular the elements of the projective system $\left(X_{\nu}, \phi_{\nu \mu}\right)$ can be taken so that for each $\nu$ the cover $X_{\nu} \rightarrow X$ is Galois. Furthermore, $\pi_{1}(X, \bar{x})=\varliminf_{\nu} \operatorname{Aut}_{X}\left(X_{\nu}\right)$.

Remark 5.9. Since for each $\nu$ the map $X_{\nu} \rightarrow X$ is finite, hence affine, it follows from [SGA 4, VII, §5] that the projective limit of schemes $\widehat{X}=$ $\lim _{\nu} X_{\nu}$ exists. Moreover, by [Mil80, Chapter III, Lemma 1.16], for any étale sheaf $\mathcal{F}$ on $X$ and for any integer $n \geq 0$ we have

$$
H_{\mathrm{et}}^{n}\left(\widehat{X}, \mathcal{F}_{\mid \widehat{X}}\right) \cong \underline{\lim }_{\longrightarrow} H_{\mathrm{et}}^{n}\left(X_{\nu}, \mathcal{F}_{\mid X_{\nu}}\right) .
$$

Corollary 5.10. $H_{\text {et }}^{1}\left(\widehat{X}, \mathcal{F}_{\mid \widehat{X}}\right)=0$ and $H_{\text {et }}^{0}\left(\widehat{X}, \mathcal{F}_{\mid \widehat{X}}\right) \cong \bigoplus_{i=1}^{r}\left(\mathbb{Z} / p^{n_{i}} \mathbb{Z}\right)^{m_{i}}$.

Proof. This is an immediate consequence of Propositions 5.6 and 5.7 and Remark 5.9.

Theorem 5.11. Let $X$ be a smooth projective connected algebraic curve defined over an algebraically closed field of characteristic $p>0$. For any closed point $\bar{x}$ of $X$ we have $\operatorname{cd}_{p}\left(\pi_{1}(X, \bar{x})\right) \leq 1$.

Proof. It follows from [Sha72, p. 55, Theorem 11] that it suffices to show that $H^{2}\left(\pi_{1}(X, \bar{x}), F\right)=0$ for any finite simple $\pi_{1}(X, \bar{x})$-module $F$ of $p$-power order. By [Mil80, p. 155-156], any such $F$ is associated uniquely to a $p$ torsion locally constant étale sheaf $\mathcal{F}$ with finite stalks. Proposition 5.4 shows that there exists $X^{\prime} \in \mathbf{F E t} / X$ such that

$$
F \cong \mathcal{F}_{\mid X^{\prime}} \cong \bigoplus_{i=1}^{r}\left(\mathbb{Z} / p^{n_{i}} \mathbb{Z}\right)^{m_{i}} .
$$

Furthermore, by [SGA 4, X, Corollary 5.2], since $X$ is a smooth projective connected algebraic curve defined over $k$, we conclude that

$$
H_{\text {et }}^{n}(X, \mathcal{F})=0 \text { for any } n \geq 2 .
$$

For every $\nu$ we consider the Hochschild-Serre spectral sequence [Mil80, p. 105, Theorem 2.20] $E_{\nu}^{r, s} \Rightarrow E^{r+s}$, where $E_{\nu}^{r, s}=H^{r}\left(\operatorname{Aut}_{X}\left(X_{\nu}\right), H_{\text {et }}^{s}\left(X_{\nu}\right.\right.$, $\left.\left.\mathcal{F}_{\mid X_{\nu}}\right)\right)$ and $E^{r+s}=H_{\text {et }}^{r+s}(X, \mathcal{F})$. Also, as in [Mil80, p. $\left.106(\mathrm{~b})\right]$, taking the projective limit we obtain a spectral sequence $E_{\infty}^{r, s} \Rightarrow E^{r+s}$, where $E_{\infty}^{r, s}=$ $H^{r}\left(\pi_{1}(X, \bar{x}), H_{\text {et }}^{s}\left(\widehat{X}, \mathcal{F}_{\mid \widehat{X}}\right)\right)$. Furthermore, it follows from [Mil80, p. 309, 
1.8] that there exists an exact sequence

$$
\begin{aligned}
0 & \rightarrow H^{1}\left(\pi_{1}(X, \bar{x}), H_{\mathrm{et}}^{0}\left(\widehat{X}, \mathcal{F}_{\mid \widehat{X}}\right)\right) \rightarrow H_{\mathrm{et}}^{1}(X, \mathcal{F}) \rightarrow H^{0}\left(\pi_{1}(X, \bar{x}), H_{\mathrm{et}}^{1}\left(\widehat{X}, \mathcal{F}_{\mid \widehat{X}}\right)\right) \\
& \rightarrow H^{2}\left(\pi_{1}(X, \bar{x}), H_{\mathrm{et}}^{0}\left(\widehat{X}, \mathcal{F}_{\mid \widehat{X}}\right)\right) \rightarrow H_{\mathrm{et}}^{2}(X, \mathcal{F}) \rightarrow H^{1}\left(\pi_{1}(X, \bar{x}), H_{\mathrm{et}}^{1}\left(\widehat{X}, \mathcal{F}_{\mid \widehat{X}}\right)\right) .
\end{aligned}
$$

Finally, we conclude from Corollary 5.10, (5.2), (5.3) and (5.4) that $H^{2}\left(\pi_{1}(X, \bar{x}), F\right)=0$. Thus, $\operatorname{cd}_{p}\left(\pi_{1}(X, \bar{x})\right) \leq 1$.

In the next two corollaries we assume that $X$ has genus $g \geq 2$. In this case it follows from [Ray82, Corollaire 4.3.2] that the $p$-Sylow subgroups of $\pi_{1}(X, \bar{x})$ are non-trivial.

Corollary 5.12. For every finite simple p-power order $\pi_{1}(X, \bar{x})$-module $F$ we have $H^{1}\left(\pi_{1}(X, \bar{x}), F\right) \cong H_{\mathrm{et}}^{1}(X, \mathcal{F})$.

Proof. The result is a consequence of Corollary 5.10 and (5.4).

Corollary 5.13. The p-Sylow subgroups of $\pi_{1}(X, \bar{x})$ are non-trivial and pro-p-free.

Proof. Recall that [Ser86, p. I-20, Proposition $14(\mathrm{i})] \operatorname{implies} \operatorname{cd}_{p}(P)=$ $\operatorname{cd}_{p}\left(\pi_{1}(X, \bar{x})\right)$, for any $p$-Sylow subgroup $P$ of $\pi_{1}(X, \bar{x})$. Moreover, it follows from Theorem 5.11 that $\operatorname{cd}_{p}\left(\pi_{1}(X, \bar{x})\right) \leq 1$. But, for a pro- $p$-group $P$ this is equivalent to $P$ being pro- $p$-free.

\section{Galois covers.}

Proof of Theorem 1.3. Let $\pi_{1}(X) \rightarrow G$ be a proper solution for the embedding problem $\left(\phi: \pi_{1}(X) \rightarrow H, G \rightarrow H\right)$. Let $Y \rightarrow X$ be the Galois $H$-cover corresponding to $\phi$. Thus, $\gamma_{\phi, \chi}=\gamma_{Y, \chi}$. Recall that $\Phi(P)$ is the Frattini subgroup of $P$ and $\mathcal{P}=P / \Phi(P)$. Observe that $\mathcal{P} \in \pi_{A}(Y)$. It follows from the correspondence described in the second paragraph of Sec-

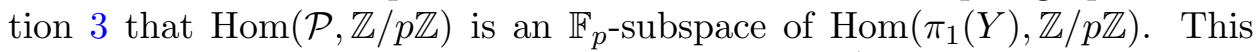
latter space is $\mathbb{F}_{p}$-isomorphic to $\operatorname{Hom}\left(\operatorname{Ker}\left(1-\mathcal{C} \mid \Omega_{Y}^{1}(0)\right), \mathbb{F}_{p}\right)$ by Serre's duality [Ser56, §9]. Therefore, (3.2) and (3.3) imply $m_{\chi} \leq t_{\chi}$, for every $\chi \in Z(H)$. Note that $\operatorname{Ker}\left(1-\mathcal{C} \mid \bigoplus_{j=0}^{l_{\chi}-1} \Omega_{Y}^{1}(0)_{\chi^{p^{j}}}\right)$ and $\operatorname{Ker}\left(1-\mathcal{C}^{l_{\chi}} \mid \Omega_{Y}^{1}(0)_{\chi}\right)$ are $\mathbb{F}_{p}[H]$-isomorphic via $\omega=\sum_{j=0}^{l_{\chi}-1} \omega_{j} \mapsto \omega_{0}$ (cf. [Pac95, Lemma 2.14]). Moreover, $\operatorname{dim}_{\mathbb{F}_{p}} \operatorname{Ker}\left(1-\mathcal{C}^{l_{\chi}} \mid \Omega_{Y}^{1}(0)_{\chi}\right)=\gamma_{Y, \chi} l_{\chi}$, therefore $t_{\chi}=\gamma_{Y, \chi} / n_{\chi}$ (cf. [Pac95, Corollary 3.6]), hence $m_{\chi} n_{\chi} \leq \gamma_{Y, \chi}$ (cf. [Ste96a, Proposition 3.4]). Conversely, suppose that $m_{\chi} \leq \gamma_{Y, \chi} / n_{\chi}$, for every $\chi \in Z(H)$. Since $t_{\chi}=\gamma_{Y, \chi} / n_{\chi}$, it follows from (3.3) there exists an $\mathbb{F}_{p}[H]$-submodule $\mathcal{B}_{\chi}$ of $\operatorname{Ker}\left(1-\mathcal{C} \mid \bigoplus_{j=0}^{l_{\chi}-1} \Omega_{Y}^{1}(0)_{\chi^{p^{j}}}\right)$ such that $\mathcal{B}_{\chi} \cong \mathcal{V}_{[\chi]}^{m_{\chi}}$. Let $\mathcal{B}=\bigoplus_{\chi \in Z(H)} \mathcal{B}_{\chi}$ and remark that there exists an $\mathbb{F}_{p}[H]$-isomorphism between $\mathcal{B}$ and $\mathcal{P}$. Once 
again by the the correspondence described in the second paragraph of Section $3, \operatorname{Hom}\left(\mathcal{B}, \mathbb{F}_{p}\right)$ is $\mathbb{F}_{p}[H]$-isomorphic to $\operatorname{Hom}(\operatorname{Gal}(Z / Y), \mathbb{Z} / p \mathbb{Z})$ for some étale cover $Z \rightarrow Y$ and $\operatorname{Gal}(Z / Y) \cong \mathcal{P}$. Therefore, Lemma 3.2 implies that $Z \rightarrow X$ is Galois and $\operatorname{Gal}(Z / X) \cong \mathcal{P} \rtimes H$. Hence $\mathcal{P} \rtimes H \in \pi_{A}(X)$. It follows from Theorem 5.11 that $\operatorname{cd}_{p}\left(\pi_{1}(X, \bar{x})\right) \leq 1$ for any closed point $\bar{x}$ of $X$. Therefore, the argument of Remark 4.4 implies that $G \in \pi_{A}(X)$.

\section{A generic condition.}

Theorem 1.3 tells us that if we are given a finite group $G$ with a normal $p$-Sylow subgroup $P$ and quotient $H$, then whether or not $G$ lies in $\pi_{A}(X)$ depends not only on the size of $P$, but also on the specific action of $H$ on $P$. The role that the action of $H$ on $P$ plays in this question was examined previously in the work of Nakajima [Nak87, Theorem A], Pacheco [Pac95, Propositions 2.4 and 2.5] and Stevenson [Ste96a, Proposition 3.5]. However, for the groups we are considering, Theorem 1.3 is stronger. In particular, it gives us a necessary and sufficient condition which is reasonably easy to compute. We begin this section with some consequences of Theorem 1.3. These involve situations where the generalized Hasse-Witt invariants can be most easily computed. At the end of this section we compute Condition A for the curve $X_{g}$ (which represents the generic geometric point of the coarse moduli scheme $\mathcal{M}_{g}$ of curves of genus $g$ ) under the assumption that $H$ is abelian. This situation is sufficient to demonstrate the strengths of our results while also distinguishing it from previous work.

As a preliminary step, we will prove the result mentioned in Remark 1.5, which deals with "ordinary Galois $H$-covers". The advantage in this case is that Condition A can be rephrased in a way that is independent of the $H$-cover. Given a finite group $G$ having a normal $p$-Sylow subgroup $P$, recall that $H=G / P, Z(H)$ denotes the set of irreducible characters $\chi$ of $H$ defined over the algebraically closed field $k$ of characteristic $p>0$ and $\chi^{0}$ is the trivial character of $H$.

Theorem 7.1. Let $G$ be a finite group having a normal $p$-Sylow subgroup $P$. Let $H=G / P$. Suppose that $\phi: \pi_{1}(X) \rightarrow H$ corresponds to a Galois $H$-cover $Y \rightarrow X$ where $Y$ is an ordinary curve. An embedding problem $\left(\phi: \pi_{1}(X) \rightarrow H, G \rightarrow H\right)$ has a proper solution if and only if $m_{\chi^{0}} \leq g$, and $m_{\chi} \leq(g-1) n_{\chi}$, for $\chi \neq \chi^{0}$.

Proof. Notice that by Remark 2.3, the Galois $H$-cover $Y$ is ordinary if and only if we have

$$
\gamma_{Y, \chi}= \begin{cases}g, & \text { if } \chi=\chi^{0} \text { and } \\ (g-1) n_{\chi}^{2}, & \text { if } \chi \neq \chi^{0}\end{cases}
$$

Thus condition A is equivalent to the condition of Theorem 7.1. 
Let $g \geq 2$ be an integer and $\pi_{A}(g)$ the set of isomorphism classes of finite groups $G$ such that $G \in \pi_{A}(X)$ for some smooth projective connected curve $X$ of genus $g$.

Remark 7.2. Suppose that there exists some smooth projective connected curve $X$ defined over $k$ such that a finite group $G \in \pi_{A}(X)$. Denote by $x \in$ $\mathcal{M}_{g}$ the point corresponding to $X$. In [Ste96, Proposition 4.2] Stevenson showed that in this case there exists an open subset $U$ of $\mathcal{M}_{g}$ containing $x$ such that for every $z \in U$ we have $G \in \pi_{A}(Z)$, where $Z$ denotes the curve corresponding to $z$. In particular, $G \in \pi_{A}\left(X_{g}\right)$, therefore $\pi_{A}\left(X_{g}\right)=\pi_{A}(g)$.

Remark 7.3. It is an immediate consequence of the definition of $\pi_{A}(g)$ that a finite group $G$ satisfying the hypothesis of Theorem 1.3 lies in $\pi_{A}(g)$ if and only if there exists a smooth projective connected curve $X$ of genus $g$ for which Condition A holds.

Notation. Let $G$ be a finite group. Denote by $d(G)$ the minimum number of generators of $G$.

Now we can prove another consequence of Theorem 1.3.

Theorem 7.4. Let $G$ be a finite group having a normal $p$-Sylow subgroup $P$. Suppose that $H=G / P$ is abelian and $g \geq 2$. A necessary and sufficient condition for $G \in \pi_{A}(g)$ is $d(H) \leq 2 g, m_{\chi^{0}} \leq g$ and $m_{\chi} \leq g-1$ for each $\chi \in Z(H)$ and $\chi \neq \chi^{0}$.

Proof. Suppose that $G \in \pi_{A}(g)$. It follows from Remark 7.3 that there exists a smooth projective connected curve $X$ and an étale Galois cover $Y \rightarrow X$ with $\operatorname{Gal}(Y / X) \cong H$ such that for every $\chi \in Z(H)$ we have $m_{\chi} \leq \gamma_{Y, \chi}$. By (2.2) we conclude that $\gamma_{Y, \chi^{0}} \leq g$ and $\gamma_{Y, \chi} \leq g-1$ for every $\chi \in Z(H)$, $\chi \neq \chi^{0}$. Moreover, since $H \in \pi_{A}(X)$, [Groth71, Corollary 2.12] implies that $d(H) \leq 2 g$. In particular, the condition of Theorem 7.4 is satisfied. Conversely, suppose that $d(H) \leq 2 g, m_{\chi^{0}} \leq g$ and $m_{\chi} \leq g-1$ for each $\chi \in Z(H)$ and $\chi \neq \chi^{0}$. Since $H$ is abelian and $d(H) \leq 2 g$, it follows from [Groth71, Corollary 2.12] that $H \in \pi_{A}\left(X_{g}\right)$, i.e., there exists an étale covering $Y_{g} \rightarrow X_{g}$ such that $\operatorname{Gal}\left(Y_{g} / X_{g}\right) \cong H$. It is a result due to Nakajima [Nak83, Theorem 2] that every étale cyclic covering $Z_{g} \rightarrow X_{g}$ of degree prime to $p$ is ordinary. (It is essential here that $X_{g}$ is generic.) This result was extended to all abelian prime to $p$ groups by Zhang [Zha92, Théorème 3.1] (again for $X_{g}$ ). Hence $Y_{g}$ is ordinary. So, by Theorem $7.1, \gamma_{Y_{g}, \chi^{0}}=g$ and $\gamma_{Y_{g}, \chi}=g-1$ for every $\chi \in Z(H), \chi \neq \chi^{0}$. Furthermore, by hypothesis, $m_{\chi^{0}} \leq g$ and $m_{\chi} \leq g-1$ for every $\chi \in Z(H), \chi \neq \chi^{0}$. Therefore, Condition $A$ holds for $X_{g}$ and by Theorem 1.3, $G \in \pi_{A}\left(X_{g}\right)$. Finally, Remark 7.2 shows that this is equivalent to $G \in \pi_{A}(g)$.

Another result in this direction is the following one from [Ste96a]. 
Theorem 7.5 ([Ste96a, Propositions 3.1 and 3.2]). Let $G$ be a finite group having a normal $p$-Sylow subgroup $P$ and $H=G / P$. Suppose that $g \geq 2$ and $d(H) \leq g$. A necessary and sufficient condition for $G \in \pi_{A}(g)$ is $m_{\chi^{0}} \leq g$ and $m_{\chi} \leq(g-1) n_{\chi}$ for each $\chi \in Z(H)$ and $\chi \neq \chi^{0}$.

Remark 7.6. Notice that for an abelian group $H$ such that $d(H) \leq 2 g$, Theorem 7.4 is stronger than Theorem 7.5 since the latter requires that $d(H) \leq g$. However, for arbitrary $H$ with $d(H) \leq g$, Theorem 7.5 is stronger than Theorem 7.4.

Now we can compare these results to a result of Nakajima. Let $G$ be a finite group, $I_{G}=\left\{\sum_{\sigma \in G} a_{\sigma} \sigma \in \mathbb{Z}[G] ; \sum_{\sigma \in G} a_{\sigma}=0\right\}$ its augmentation ideal and $t(G)$ the minimum number of generators of $I_{G}$. Suppose that there exists a smooth projective curve $X$ of genus $g$ such that $G \in \pi_{A}(X)$, i.e., $G \cong \operatorname{Gal}(Y / X)$ for some étale Galois cover $Y \rightarrow X$.

Theorem 7.7 (Nakajima, [Nak84, Theorem 4]). There exists a short exact sequence of $k[G]$-modules

$$
1 \rightarrow \Omega_{Y}^{1}(0) \rightarrow k[G]^{g} \rightarrow I_{G} \rightarrow 1 .
$$

Corollary 7.8 (Nakajima, [Nak87, Theorem A]). $t(G) \leq g$.

Notation. We call Condition B the inequality of Corollary 7.8.

Remark 7.9. From the definition of $\pi_{A}(g)$, Theorem 7.7 and Corollary 7.8, we see that Condition B is necessary for $G \in \pi_{A}(g)$.

Corollary 7.10. Let $G$ be a finite group having a normal $p$-Sylow subgroup $P, H=G / P$. Suppose that either: (a) $H$ is abelian and $d(H) \leq 2 g$; or (b) $d(H) \leq g$. Under either hypothesis (a) or (b) Condition $\mathrm{A}$ is equivalent to Condition B.

Proof. By [Ste96a, Proposition 3.5] Condition A implies Condition B without any restrictions on $H$. Conversely, by [Ste96, Proposition 3.1] Condition B implies that $m_{\chi^{0}} \leq g$ and $m_{\chi} \leq(g-1) n_{\chi}$ for each $\chi \in Z(H)$ and $\chi \neq \chi^{0}$. Under hypothesis (a) (resp. (b)) Theorem 7.4 (resp. 7.5) show that the latter condition implies that $G \in \pi_{A}(g)$. Now by Theorem 1.3 this implies Condition A.

In order to obtain a converse in the case where $H$ is a non-abelian finite quotient of $\Gamma_{g}$ we need to generalize the Nakajima-Zhang result ([Nak83, Theorem 2] and [Zha92, Théorème 3.1]) to non-abelian Galois étale covers of degree prime to $p$ of $X_{g}$. Another option is to show that there exists an ordinary Galois $H$-cover of a curve $X$ of genus $g$ and apply [Ste96] (cf. Remark 1.5). Very recently M. Raynaud has found a counter example to both these approaches. 
Example 7.11. Theorem 7.4 gives a result which is not covered by [Ste96a, Theorem 3.2] in the case where $H$ is abelian and $g<d(H) \leq 2 g$. Let $n \geq 1$ be an integer and let $g \geq 2$ be an integer. Let $H=(\mathbb{Z} / n \mathbb{Z})^{2 g}$ and label the elements $\tau_{j}$ for $j=1, \ldots, n^{2 g}$. For each $i=1,2, \ldots, g-1$ let $P_{i}=(\mathbb{Z} / p \mathbb{Z})^{n^{2 g}}$ and $P_{g}=\mathbb{Z} / p \mathbb{Z}$. Pick a basis $a_{i, \tau_{1}}, \ldots, a_{i, \tau_{n}{ }^{2 g}}$ for $P_{i}$ for $i=1, \ldots, g-1$ and let $a_{g}$ be a basis of $P_{g}$. Then we define an action of $H$ on each $P_{i}$ for $i=1, \ldots, g-1$ as follows: $\rho_{i}: H \rightarrow \operatorname{Aut}\left(P_{i}\right)$ by $\rho_{i}\left(\tau_{j}\right) a_{i, \tau_{l}}=a_{i, \tau_{j} \tau_{l}}$. With this action each $P_{i}$ is isomorphic to $\mathbb{F}_{p}[H]$, which is the $H$-module defined over $\mathbb{F}_{p}$ corresponding to the regular representation of $H$. Let $H$ act on $P_{g}$ trivially. Now let $P=\bigoplus_{i=1}^{g} P_{i}$ with the induced action of $H$ on $P$. Then $P$ is isomorphic to $(\mathbb{Z} / p \mathbb{Z})^{n^{2 g}(g-1)+1}$ as a group and to $\mathbb{F}_{p}[H]^{g-1} \oplus \mathbb{F}_{p}$ as an $\mathbb{F}_{p}[H]$-module. Let $G$ be defined as the semi-direct product $P \rtimes H$ with respect to this action.

By construction $P \otimes_{\mathbb{F}_{p}} k$ is isomorphic as a $k[H]$-module to $k[H]^{g-1} \oplus k$. Let $Z(H)$ be the set of irreducible characters of $H$ defined over $k$ and let $\chi^{0}$ be the trivial character of $H$. Then using the notation of Section 1.1, $m_{\chi^{0}}=g$ and $m_{\chi}=g-1$ for $\chi \neq \chi^{0}$. Note that since $H$ is abelian, by Zhang's theorem [Zha92, Théorème 3.1], any Galois $H$-cover $Y_{g}$ of $X_{g}$ is ordinary, thus $\gamma_{Y_{g}, \chi^{0}}=g$, and $\gamma_{Y_{g}, \chi}=g-1$ for $\chi \neq \chi^{0}$. In particular, Condition A is satisfied for the curve $X_{g}$, therefore $G \in \pi_{A}\left(X_{g}\right)=\pi_{A}(g)$.

Remark 7.12. In the set-up of Example 7.11, as the rank of $H$ is greater than $g$, Theorem 7.5 does not apply. If we keep $P$ the same but change the action of $H$ on $P$ in any way, then $G$ will not lie in $\pi_{A}(g)$, because for some character $\chi \neq \chi^{0}$ we would have $m_{\chi}>g-1$ or $m_{\chi^{0}}>g$. Finally, if we replace $P$ by any $p$-group $Q$ with Frattini quotient isomorphic to $P$ and extend the action of $H$ in $P$ of Example 7.11 to an action of $H$ in $Q$, then we get (as in the end of the proof of Theorem 1.3) $Q \rtimes H \in \pi_{A}\left(X_{g}\right)=\pi_{A}(g)$.

\section{References}

[CheWei34] C. Chevalley and A. Weil, Über das Verhalten der Integrale 1. Gattung bei Automorphismen des Funktionenkörpers, A. Weil Collected Papers, I, 68-71.

[Groth71] A. Grothendieck, Rêvetements étales et groupe fondamental (SGA 1), Lecture Notes in Math., 244, Springer-Verlag, 1971.

[SGA 4] A. Grothendieck, M. Artin and J.-L. Verdier, SGA 4: Théorie des topos et cohomologie étale des schémas, Lecture Notes in Mathematics, 269, 270 and 305, Springer-Verlag, 1972-1973.

[Har95] D. Harbater, Fundamental groups and embedding problems in characteristic $p$, Contemporary Mathematics, 186 (1995), 353-369.

[Isa76] I.M. Isaacs, Character Theory of Finite Groups, Academic Press, 1976.

[Kat88] N. Katz, Local-to-global extensions of representations of fundamental groups, Ann. Inst. Fourier, 36 (1986), 69-106. 
[Mil80] J.S. Milne, Étale cohomology, Princeton University Press, 1980.

[Pac95] A. Pacheco, Unramified Galois coverings of algebraic curves, J. Number Theory, 53 (1995), 211-228.

[Nak83] S. Nakajima, On generalized Hasse-Witt invariants of an algebraic curve, Adv. Studies Pure Math., 2 (1983), 69-88.

[Nak84]_ On the Galois module structure of the cohomology groups of an algebraic variety, Invent. Math., 75 (1984), 1-8.

[Nak87] On the quotients of the fundamental group of an algebraic curve, Adv. Stu. Pure Math., 12 (1987), 259-264.

[Ray82] M. Raynaud, Sections des fibrés véctoriels sur une courbe, Bull. Soc. Math. France, 110 (1982), 103-125.

[Ruc86] H.G. Rück, L-series and class groups of function fields, J. Number Theory, 22 (1986), 177-189.

[Ser56] J.-P. Serre, Sur la topologie des variétés algébriques en caractéristique p, in 'Symp. Topologia Algebraica' (Mexico City), 1956, 24-53.

[Ser86] _ Cohomologie Galoisienne, Lecture Notes in Mathematics, 5 (1986), Springer-Verlag.

[Ser88]_ _ Algebraic Groups and Class Fields, Springer-Verlag, 1988.

[Ser90] Constructions de revêtements étales de la droite affine en caractéristique p, C. R. Acad. Sci. Paris, 311, Série I, (1990), 341-346.

[Sha72] S.S. Shatz, Profinite Groups, Arithmetic, and Geometry, Princeton University Press, 1977.

[Ste96] K. Stevenson, Galois groups of étale covers of projective curves in characteristic p, J. Algebra, 182 (1996), 770-804.

[Ste96a] Conditions related to $\pi_{1}$ of projective curves, J. Number Theory, (March, 1998), 62-79.

[Zha92] B. Zhang, Revêtements étales abéliens de courbes génériques et ordinarité, Ann. Fac. Sci. Toulouse Sér. 6, 1 (1992), 133-138.

Received May 18, 1998. Amílcar Pacheco was partially supported by CNPq research grant number 300896/91-3 and Pronex, Brazil. Section 5 was partially written during a visit of Amílcar Pacheco to the University of Campinas, São Paulo, Brazil. He would like to thank this Institution for its hospitality and Antônio Engler for many conversations. Katherine F. Stevenson was supported by NSA grant MDA 9049710017. Both authors would like to thank David Harbater and his student Rachel Pries for their careful reading and many suggestions on previous versions of this paper.

Universidade Federal do Rio de Janeiro

RIO DE JANEIRO RJ

BRASIL

E-mail address: amilcar@impa.br

California Institute of Technology

Pasadena, CA 91125

E-mail address: kfs@vision.caltech.edu 